\title{
Role of CT scan in modern Neurosurgery.
}

Balgopal Karmacharya

Associate Professor and Chief, Department of Neurosurgery, Manipal Teaching Hospital, Pokhara, Nepal.

Email: dr.balgopal@hotmail.com

This year we are celebrating the half centenary of the discovery and clinical application of computed tomography (CT) in medicine. CT scan was started in 1971. The first CT scan on a live patient took place in September 1971 at Atkinson Morley's Hospital in London. Sir Godfrey Hounsfield and Allan M Cormack received the Nobel Prize for their significant discovery. ${ }^{1}$

Since its development, CT scan has been used virtually in every physical medical discipline in one or the other form. In trauma it is used for fast, accurate, precise determination of the extent of injury from head to toe, even in unstable patients. In oncology, it is used for detection and staging of different cancers and for documenting their response to treatment. CT is nothing short of a game changer in medicine. Exploratory surgery is largely eliminated because of the availability of CT scan. Similarly, in internal medicine, general surgery, cardiology, cardiothoracic and vascular surgery, orthopedics, pediatrics, Ophthalmology, Gynecology; you name it and CT scan is used in that specialty uses CT scan for detection of pathology, its severity and for monitoring treatment outcome. And we have come a long way since the first clinical application of this beautiful technology.

With time, we are having ever faster scanning, higher spatial resolution and faster data reconstruction and falling radiation dose that has increased its applications in all specialties. As with other applications, we have made great strides in its application for neurological problems relating to the brain and spine.

CT scan is an indispensable imaging modality for diseases of the brain and spine. CT scan is widely used in trauma, tumor, infective and inflammatory conditions, hydrocephalus, and acute ischemic and hemorrhagic stroke of the brain. Its use has helped us diagnose neurological and neurosurgical diseases fast and precisely and save lives.

Newer applications include- perfusion scan and CT angiography in acute ischemic stroke, to determine the volume of brain at risk and also to determine the blocked cerebral arteries. ${ }^{2}$ Another application is CT-PET to determine the origin of seizure focus in patients with epilepsy and to document multiple or metastatic lesions in cases of secondary cancers of the brain. ${ }^{3,4} \mathrm{CT}$ angiography has almost replaced diagnostic digital subtraction angiography for diagnosis of different vascular lesions of the brain and spine. Time-resolved 4D-CT angiography has been found to have high diagnostic yield for intracranial arteriovenous shunts.

The future of CT imaging looks even better. With the development of artificial intelligence, 3D printing and cinematic rendering, CT will not only help in making diagnosis but also help us predict the patient outcome.

\section{Refrences:}

1. Bhattacharyya KB. Godfrey Newbold Hounsfield (19192004): The man who revolutionized neuroimaging. Ann Indian Acad Neurol. 2016 Oct-Dec;19(4):448-450. https://doi.org/10.4103/0972-2327.194414.

2. Heit JJ, Wintermark M. Perfusion Computed Tomography for the Evaluation of Acute Ischemic Stroke: Strengths and Pitfalls. Stroke. 2016;47(4):1153-8. https://doi.org/10.1161/STROKEAHA.116.011873.

3. von Oertzen TJ. PET and ictal SPECT can be helpful for localizing epileptic foci. Curr Opin Neurol. 2018;31(2):184-191.

https://doi.org/10.1097/WCO.0000000000000527.

4. Galldiks N, Lohmann P, Albert NL, Tonn JC, Langen KJ. Current status of PET imaging in neuro-oncology. Neurooncol Adv. 2019;1(1):vdz010. https://doi.org/10.1093/noajnl/vdz010. 